\title{
Titanium Kirschner Wires Resist Biofilms Better Than Stainless Steel and Hydroxyapatite-coated Wires: An In Vitro Study
}

\author{
James P McEvoy ${ }^{1}$, Philip Martin ${ }^{2}$, Arshad Khaleel ${ }^{3}$, Shobana Dissanayeke ${ }^{4}$
}

\begin{abstract}
Aim: External fixation surgery is frequently complicated by percutaneous pin site infection focused on the surface of the fixator pin. The primary aim of this study was to compare biofilm growth of clinically isolated pin site bacteria on Kirschner wires of different materials.

Materials and methods: Two commonly infecting species, Staphylococcus epidermidis and Proteus mirabilis, were isolated from patients' pin sites. A stirred batch bioreactor was used to grow these bacteria as single culture and co-cultured biofilms on Kirschner wires made of three different materials: stainless steel, hydroxyapatite-coated steel and titanium alloy.

Results: We found that the surface density of viable cells within these biofilms was $3 \mathrm{x}$ higher on stainless steel and $4.5 \mathrm{x}$ higher on hydroxyapatitecoated wires than on the titanium wires.

Conclusion: Our results suggest that the lower rates of clinical pin site infection seen with titanium Kirschner wires are due to, at least in part, titanium's better bacterial biofilm resistance.

Clinical significance: Our results are consistent with clinical studies which have found that pin site infection rates are reduced by the use of titanium relative to stainless steel or hydroxyapatite-coated pins.

Keywords: Bacterial adhesion, Biofilms, External fixation, Infection, Orthopedics.

Strategies in Trauma and Limb Reconstruction (2019): 10.5005/jp-journals-10080-1426
\end{abstract}

\section{INTRODUCTION}

External fixation is used widely to treat bone fractures and as a technique in limb reconstruction. ${ }^{1}$ Metal pins known as Kirschner wires, or K-wires, are implanted into the bone above and below a fracture and an external frame is attached, fixing the bone segments in place to allow effective bone union. ${ }^{2}$ External fixation is used for traumatic fractures which is, in itself, a leading cause of disease burden worldwide. ${ }^{3}$ The number of external fixation procedures is increasing in many developed countries, coincident with an aging population and a rise in the frequency of fractures in geriatric patients. ${ }^{4}$

Pin sites (also known as "pin tracks") are prone to infection. ${ }^{5}$ The infection rate varies widely between studies; up to $100 \%$ in some animal models ${ }^{6}$ and clinical studies, ${ }^{7}$ with the majority of published clinical estimates being closer to $50 \% .{ }^{8}$ Infection rates remain high even with antibiotic prophylaxis and regular pin site cleaning with topical antiseptics ${ }^{9}$ and are further exacerbated by comorbidities such as diabetes. ${ }^{10}$ Pin site infections are treated in the first instance with systemic antibiotics and in the last resort by removing the infected pins. ${ }^{9}$ Chronic infection may lead to pin loosening and accompanying loss of bone alignment and, in rare cases, to osteomyelitis and bacteraemia. ${ }^{7}$

Pin site bacteria are part of the commensal skin flora which become opportunistic pathogens within the wound. ${ }^{11}$ Staphylococci are most commonly implicated, with S. aureus and S. epidermis accounting for the majority of infections. ${ }^{7,12}$ Gram-negative bacteria such as Escherichia coli, P. mirabilis, and Pseudomonas aeruginosa are also found commonly. ${ }^{13}$

Bacterial biofilms on the surface of pins act as the focus of infection. ${ }^{14}$ Biofilms form when free-floating, planktonic bacteria attach to solid surfaces using flagella or fimbriae. ${ }^{15}$ Adhesion
1,2,4 Department of Biological Sciences, Royal Holloway, University of London, Egham, Surrey, UK

${ }^{3}$ Rowley Bristow Orthopaedic Unit, Ashford and St Peter's Hospitals NHS Foundation Trust, Chertsey, Surrey, UK

Corresponding Author: James P McEvoy, Department of Biological Sciences, Royal Holloway, University of London, Egham, Surrey, UK, Phone: +44 7759236692, e-mail: james.mcevoy@rhul.ac.uk

How to cite this article: McEvoy JP, Martin P, Khaleel A, et al. Titanium Kirschner Wires Resist Biofilms Better Than Stainless Steel and Hydroxyapatite-coated Wires: An In Vitro Study. Strategies Trauma Limb Reconstr 2019;14(2):57-64.

Source of support: Nil

Conflict of interest: None

is followed by bacterial growth and secretion of exopolymeric matrix substances, mostly polysaccharides, which stick bacterial cells to one another and to the colonized surface. ${ }^{15}$ These strongly surface-associated communities allow bacteria to survive both the host immune system and clinical interventions such as antibiotic treatment. ${ }^{11}$ The ease with which a particular bacterial strain forms a biofilm depends on the material surface; the physical characteristics (e.g., roughness) and chemical nature (e.g., hydrophobicity/ hydrophilicity) have both been found to determine susceptibility to biofilm growth. ${ }^{16}$

Following improvements in perioperative sterility and postoperative pin site care, ${ }^{17}$ one strategy for reducing infection rates further is to use pins which are biofilm resistant or otherwise antimicrobial. ${ }^{18}$ The pin materials in common use are titanium alloys and stainless steel, with or without a hydroxyapatite coating. ${ }^{18}$ Most 
clinical studies have found titanium pins are less often infected than uncoated stainless steel pins, ${ }^{19-21}$ although some researchers have found no significant difference. ${ }^{5}$ In animal models, some studies have detected lower infection rates with titanium than with stainless steel ${ }^{22}$ and in others a small or nonsignificant difference. ${ }^{23}$ In vitro studies on bacterial adhesion are ambiguous, with studies mostly on staphylococcal species failing to show a consistent preference for either material. ${ }^{24}$

Hydroxyapatite-coated pins have been shown unequivocally to improve bone contact through osseointegration which, in turn, reduces the frequency of pin loosening in both humans and animals. ${ }^{25}$ It is unclear, however, whether the improved bone contact made by hydroxyapatite-coated pins reduces clinical infection rates, ${ }^{26}$ despite an in vitro study showing decreased staphylococcal adherence to such pins. ${ }^{27}$ The interpretation of in vitro studies in this area has been complicated by variation in important factors such as the bacterial species and strains selected, biofilm definition and measurement, alloy composition, and the surface treatment of the metal.

In this study, clinically interpretable in vitro biofilm data were obtained by first isolating and identifying clinical pin site bacteria and then growing selected clinical strains as biofilms on as-received, commercially available K-wires made from titanium alloy, uncoated stainless steel, and hydroxyapatite-coated steel. A bioreactor was used to process samples in parallel, ensuring identical biofilm growth conditions for an accurate comparison of viable (and potentially pathogenic) cell density. The use of patient-derived bacterial strains and untreated commercially available K-wires provides added practical relevance on pin site infection. The study's main finding that titanium resists biofilm growth better than the other two helps to explain the superior clinical outcomes reported for this metal.

\section{Materials and Methods Clinical Bacteria Collection}

Bacteria were isolated from pin site swabs taken from patients with lower limb external fixation devices at St. Peter's Hospital, Surrey, UK. A swab of each pin site at the clinically uninfected wire or wound interface (4-5 per patient) was taken from three patients and transported in Amies transport medium to the laboratory. The swabs were then transferred into phosphate-buffered saline (PBS, $\mathrm{pH}$ 7.5) and incubated overnight at $37.5^{\circ} \mathrm{C}$. Each swab was then removed from its solution, and the overnight cultures were mixed with glycerol $(150 \mu \mathrm{L} / \mathrm{mL})$. These glycerol stocks were stored at $-80^{\circ} \mathrm{C}$ until required.

\section{Clinical Bacteria Isolation}

Bacteria were isolated on three types of agars (Oxoid): eosin methyl blue agar, mannitol salt agar, and sheep blood agar. Clinically isolated culture stocks were plated onto each type of agar, and distinct colonies were assessed by gram staining and light microscopy. Distinction criteria were based on colony form, margin, elevation, differing hemolysis, mannitol fermentation, and type of agar. Isolated colonies were incubated in liquid growth medium overnight, then mixed with glycerol $(150 \mu \mathrm{L} / \mathrm{mL})$, and stored at $-80^{\circ} \mathrm{C}$ until required.

\section{Extraction and Amplification of Bacterial DNA}

DNA was extracted from the bacterial isolates using standard techniques ${ }^{28}$ and amplified with nested polymerase chain reaction (nPCR). ${ }^{29,30}$ The $n P C R$ products were assessed using ethidium bromide in a $1.8 \%$ agarose gel subjected to electrophoresis at $100 \mathrm{~V}, 200 \mathrm{~mA}, 100 \mathrm{~W}$ for 45 minutes. Amplicons of approximately $700 \mathrm{bp}$ [outer primer (OP)] and $300 \mathrm{bp}$ [inner primer (IP)] were expected and compared against a 100-bp ladder (New England Biolabs: Hertfordshire, UK). The nPCR products were sequenced (Eurofins: Wolverhampton, UK), and the nucleotide sequences were analyzed using the online nucleotide basic local alignment search tool, BLAST. ${ }^{31}$

\section{Microtiter Plate Assay}

A microtiter plate assay was used to measure the biofilm-forming ability of each clinical isolate. ${ }^{32}$ Five microliters of overnight culture was pipetted into $1 \mathrm{~mL}$ lysogeny broth (LB) in 24-well plates. Cultures were incubated at $37.5^{\circ} \mathrm{C}$ for 16 hours with agitation at $160 \mathrm{rpm}$ and incubated for a further 24 hours without agitation to allow biofilms to settle. The growth medium was pipetted out and the wells washed three times with PBS to remove loose cells that were not attached to the biofilm. Wells were stained with $1 \%$ crystal violet solution for 5 minutes and washed three times with PBS to remove any residual dye. Two milliliters of $95 \%$ ethanol was used to solubilize the stain and absorbance was read at $600 \mathrm{~nm}$ on a SpectraMax 190 plate reader (Molecular Devices: Berkshire, UK) to measure biofilm growth in each well. All isolates were grown in parallel with a strong biofilm former (E. coli Nissle 1917) and a weak one (E. coli DH10B) as a positive and negative control, respectively.

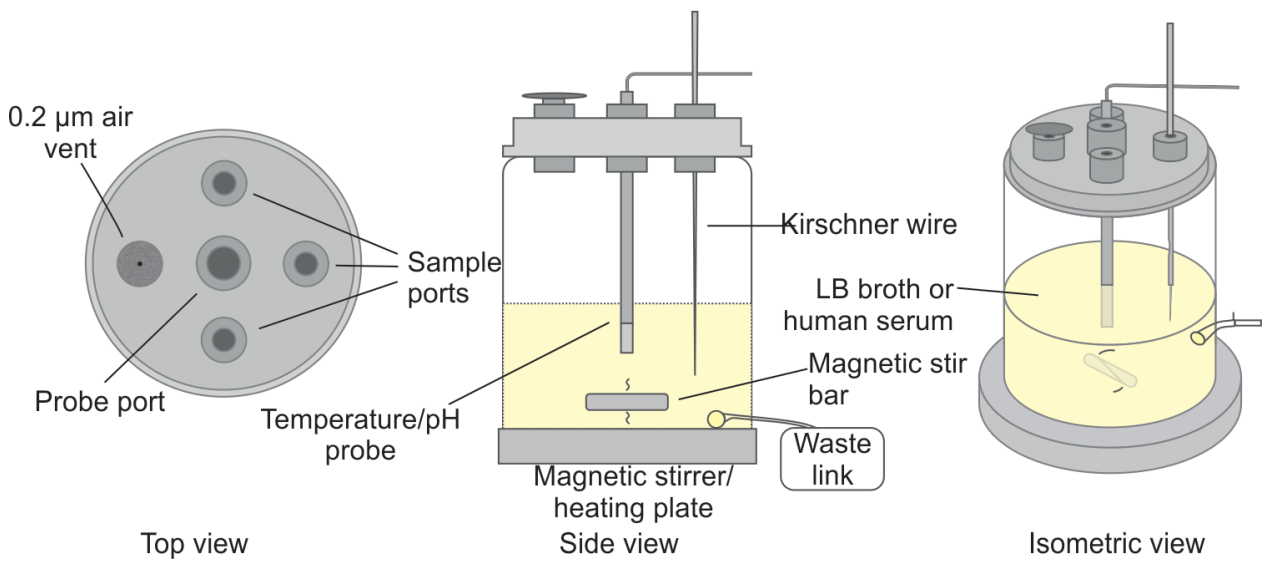

Fig. 1: Schematic diagram of the stirred batch tank bioreactor used to grow biofilms on orthopedic K-wires 


\section{Bioreactor Biofilm Growth}

A stirred-tank batch bioreactor (Fig. 1), adapted for use with 1.8-mm orthopedic K-wires, was built in-house. Its design was based on that of the CDC Biofilm Reactor (Biosurface Technologies Corporation: Bozeman, Montana, USA) $)^{33}$ and was built from highdensity polyethylene, polypropylene, and polycarbonate to allow for autoclaving between batches. Growth media was stirred and maintained at $37.5^{\circ} \mathrm{C}\left( \pm 2^{\circ} \mathrm{C}\right)$ using a heated magnetic stir plate (VWR: Leicestershire, UK) set at $180 \mathrm{rpm}$. A pH electrode and meter (Mettler Toledo: Leicester, UK) were used to continuously monitor the temperature and $\mathrm{pH}$ of the growth medium, which was either $10 \%$ diluted LB broth (Sigma-Aldrich: Dorset, UK) or human serum (TCS Biosciences, Bucks, UK). Biofilms were grown on orthopedic wires inserted into the bioreactor for 48 hours from the point of inoculation of the growth medium. A waste pipe/nutrient replacement system allowed users to remove waste products and to replace nutrients in a sterile manner. The insertion of K-wires and nutrients and the removal of wastes took place in a class II biosafety cabinet under sterile conditions.

\section{Kirschner Wires}

Uncoated 316L stainless steel K-wires were obtained from De Soutter Medical (Bucks, UK), Ti-6Al-4V titanium alloy K-wires were obtained from JPP Management (Scionzier, France) and hydroxyapatite-coated steel K-wires were obtained from Ortho Solutions (Essex, UK). Wires were autoclaved before they were positioned in the bioreactor.

\section{Fluorescent Microscopy of Biofilms}

Biofilms for visualization were grown in the bioreactor on $22 \mathrm{~mm} \times$ $70 \mathrm{~mm}$ glass coverslips, immersed in LB growth medium, and held in place by a custom-built coverslip holder. After 48 hours of biofilm growth, the coverslips were removed, washed in PBS, and fixed in $95 \%$ methanol for 10 minutes. Fixed slides were then stained using Invitrogen filmtracer ${ }^{\circledR}$ SPYRO ruby biofilm matrix stain (Thermo Fisher Scientific: Hertfordshire, UK) according to the manufacturer's instructions. Coverslips were attached to a glass slide using glue with white tack (UHU) around the edges of the coverslip to elevate it and preserve the three-dimensional structure of the biofilm. A

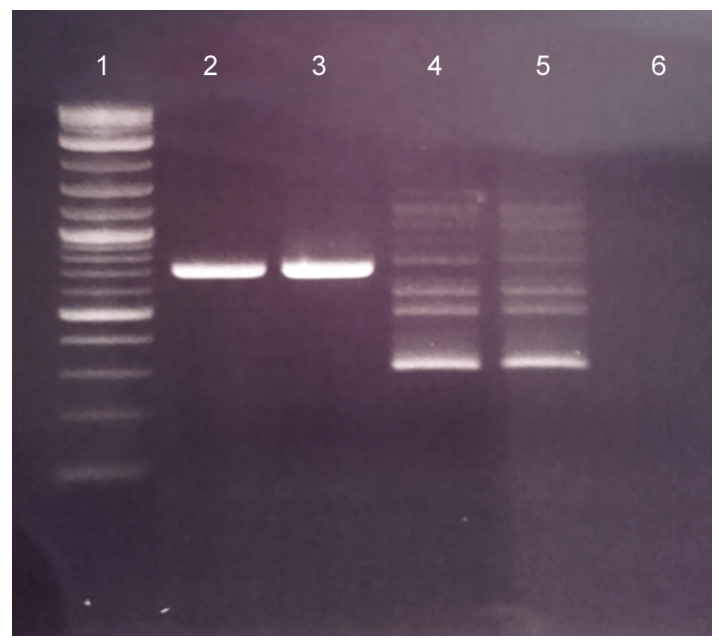

Fig. 2: Agarose gel electrophoresis of the nPCR products. (1) 100-bp ladder; (2) OP with S. epidermidis DNA; (3) OP with P. mirabilis DNA; (4) IP with S. epidermidis DNA; (5) IP with P. mirabilis DNA; and (6) Negative control
Nikon Eclipse Ti-E fluorescent microscope with a mercury lamp set at $450 \mathrm{~nm}$ was used to excite the stain and visualize the biofilms.

\section{Comparison of Biofilm Formation on Orthopedic Materials}

Biofilms were grown inside the bioreactor (see Fig. 1) on 1.8-mm-diameter K-wires in 10\% LB diluted with PBS. This medium was replaced by undiluted human serum for some experiments. To efficiently detach the biofilms from the K-wires, ${ }^{34} 4 \mathrm{~mL}$ PBS was pipetted into a $20 \mathrm{~mL}$ test tube, and the biofilms were dispersed into the PBS from the surface of the K-wires by ultrasound treatment. The K-wires were then immersed in $1 \%$ crystal violet solution for 10 minutes and examined under $55 \times$ magnification to confirm the complete removal of the biofilm from the pin. Sonicated hydroxyapatite-coated K-wires were checked under $55 \times$ magnification without staining because the crystal violet stained the coating, making any biofilms present indistinguishable from the K-wire. Loss of the hydroxyapatite coating during sonication was addressed by cutting the end of the wire after each round of sonication to produce a fresh surface for the next experiment.

A drop plate method was adapted to assess the number of viable cells. ${ }^{35}$ The surface density of viable cells in the biofilms was calculated using the formula: viable cell surface density $\left(\mathrm{CFU} / \mathrm{cm}^{2}\right)$ $=\log _{10}[$ (mean colony count/drop volume) (10 dilution) (PBS volume/ $S A)$ ], where $S A=$ colonized surface area of the K-wire. The surface area was calculated in turn using the formula $S A=2 \pi r d+\pi r^{2}$, where $d=$ immersion depth $(\mathrm{cm})$ and $r=\mathrm{K}$-wire radius $(\mathrm{cm})$.

\section{Statistical Methods}

SPSS Statistics 21.0 (IBM: Armonk, New York, USA) was used to analyze the microbiological results which were represented graphically as mean values \pm standard error. One-way analysis of variance tests were used to evaluate the difference between groups.

\section{Results}

\section{Bacterial Identification}

The $\mathrm{nPCR}$ products were examined by agarose gel electrophoresis (Figs 2 and 3). Amplicons of $\sim 709$ and 287 bp in size were observed, equating to the OP and IP, respectively. The PCR products were, therefore, of the expected size, and no DNA contamination was

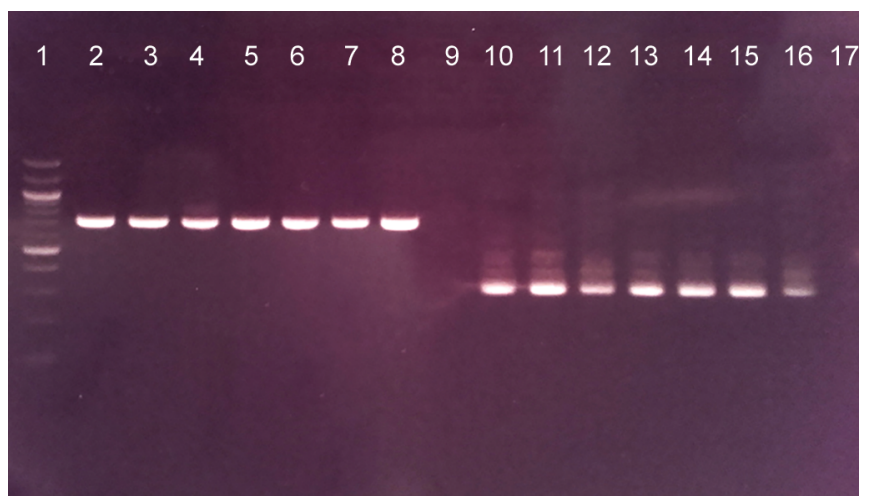

Fig. 3: Agarose gel electrophoresis of the nPCR products by species. (1) 100-bp ladder; (2) OP S. epidermidis; (3) OP S. epidermidis; (4) OP S. epidermidis; (5) OP S. aureus; (6) OP S. aureus; (7) OP P. mirabilis; (8) OP P. mirabilis; (9) OP negative control; (10) IP S. epidermidis; (11) IP S. epidermidis; (12) IP S. epidermidis; (13) IP S. aureus; (14) IP S. aureus; (15) IP P. mirabilis; (16) IP P. mirabilis; and (17) IP negative control 
Table 1: BLAST results of bacterial isolates

\begin{tabular}{|c|c|c|c|c|}
\hline $\begin{array}{l}\text { Closest } \\
\text { species match }\end{array}$ & E-value & Identity (\%) & Number of nucleotides & Sequence \\
\hline S. aureus & $2^{-130}$ & 100 & 257 & $\begin{array}{l}\text { ATCTTGACATCCTTTGACAACTCTAGAGATAGAGCCTTCCCCTTCGGGG- } \\
\text { GACAAAGTGCAGGTGGTGCATGGTTGTCGTCAGCTCGTGTCGTGAGAT- } \\
\text { GTTGGGTTAAGTCCCGCAACGAGCGCAACCCTTAAGCTTAGTTGCCAT- } \\
\text { CATTAAGTTGGGCACTCTAAGTTGACTGCCGGTGACAAACCGGAGGAA- } \\
\text { GGTGGGGATGACGTCAAATCATCATGCCCCTTATGATTTGGGCTACACACGT- } \\
\text { GCTACAATGGA }\end{array}$ \\
\hline S. epidermidis & $5^{-131}$ & 99 & 265 & $\begin{array}{l}\text { ATCTTGACATCCTCTGACCCCTCTAGAGATAGAGTTTTCCCCTTCGGGG- } \\
\text { GACAGAGTGACAGGTGGTGCATGGTTGTCGTCAGCTCGTGTCGTGAGA- } \\
\text { TGTTGGGTTAAGTCCCGCAACGAGCGCAACCCTTAAGCTTAGTTGCCAT- } \\
\text { CATTAAGTTGGGCACTCTAAGTTGACTGCCGGTGACAAACCGGAGGAA- } \\
\text { GGTGGGGATGACGTCAAATCATCATGCCCCTTATGATTTGGGCTACACACGT- } \\
\text { GCTACAATGGACTTACAAT }\end{array}$ \\
\hline P. mirabilis & $8^{-134}$ & 100 & 272 & $\begin{array}{l}\text { TTACCTACTCTTGACATCCAGCGAATCCTTTAGAGATAGAGGAGTGC- } \\
\text { CTTCGGGAACGCTGAGACAGGTGCTGCATGGCTGTCGTCAGCTCGTGTT- } \\
\text { GTGAAATGTTGGGTTAAGTCCCGCAACGAGCGCAACCCTTATCCTTTGTT- } \\
\text { GCCAGCACGTGATGGTGGGAACTCAAAGGAGACTGCCGGTGATAAACCG- } \\
\text { GAGGAAGGTGGGGATGACGTCAAGTCATCATGGCCCTTACGAGTAGGGCTA- } \\
\text { CACACGTGCTACAATGGATCAATCTC }\end{array}$ \\
\hline
\end{tabular}

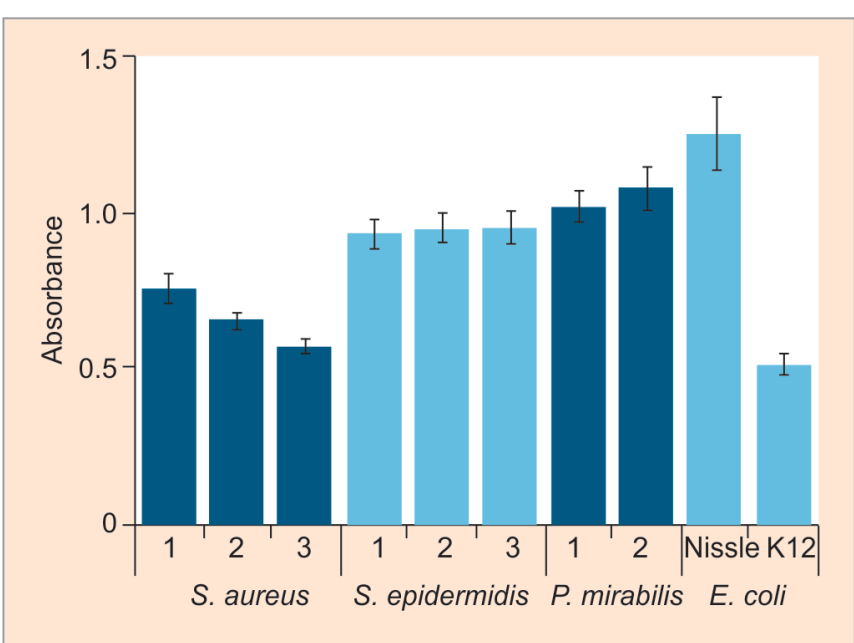

Fig. 4: Microtiter plate assay of biofilm formed by isolates, compared with controls. Absorbance of the crystal violet stain was measured at $600 \mathrm{~nm}$. The columns represent the means of the four repeats with error bars representing \pm standard error

observed in the negative controls. Species identity was assigned to gel lanes once sequencing has been completed.

The BLAST results of the nPCR products are shown in Table 1. Three species commonly associated with wound infections were identified: S. aureus, S. epidermidis, and P. mirabilis. All sequences exhibited $E$-values far below the confidence threshold of $10^{-5}$, indicating a very low probability of a random match.

\section{Microtiter Plate Assay of Biofilm Growth}

Figure 4 shows the results of the microtiter assay from each clinical isolate; each assay comprised four repeats per 24-well plate, and the assay was repeated four times. All of the clinical isolates grew as biofilms on the plates, and P. mirabilis and S. epidermidis formed biofilms almost as well as the positive control E. coli Nissle 1917. These two species were taken forward for further investigation. The three $S$. aureus isolates showed less biofilm growth than $S$. epidermidis, in line with previous results. ${ }^{36}$

\section{Fluorescent Microscopy of Biofilms}

Figure 5 shows epifluorescent microscopic images of co-cultured biofilms of $P$. mirabilis and S. epidermidis on glass coverslips. Both spherical S. epidermidis (green circles) and swarming rod-shaped $P$. mirabilis (yellow circles) are visible within the same biofilm. The heterogeneous structure typical of bacterial biofilms is apparent, with clusters of cells separated by large voids in which no cells are present. $^{37}$

\section{Comparison of Biofilm Growth on K-wires}

Biofilms of patient-isolated S. epidermidis and P. mirabilis, both separately and in co-culture, were grown in the bioreactor on $\mathrm{K}$-wires made from different materials. Figure 6 shows that titanium alloy showed less biofilm formation than stainless steel and hydroxyapatite-coated steel under all conditions tested. Titanium's relative resistance to biofilm growth across all our experiments (Fig. $6 \mathrm{D}$ ) equates to a $4.5 \times$ decrease in biofilm growth on titanium relative to hydroxyapatite and a $3.0 \times$ decrease relative to stainless steel. $P$. mirabilis biofilms (Fig. 6B) grew slightly better than S. epidermidis biofilms (Fig. 6A), a result which is consistent with our microtiter plate assay. Biofilms in human serum (Fig. 6C) grew better than in diluted LB medium (Figs $6 \mathrm{~A}$ and B). Co-cultured biofilms in human serum (Fig. 6C) grew no better than biofilms of $P$. mirabilis alone (data not shown).

\section{Discussion}

Bacterial biofilm formation on the surface of orthopedic pins allows infections to develop and persist. ${ }^{38}$ Appropriately chosen pin materials could, therefore, be used to prevent biofilm formation and thereby reduce infection rates. ${ }^{18}$ In vivo clinical studies are clearly required to compare surgical infection rates and to inform practice. Such experiments, however, cannot easily reveal the contribution of biofilm growth to infection because the developing biofilms are hidden under the patient's skin, and the biofilms are likely to be disturbed as the pins are removed. ${ }^{39}$ By using a physiologically relevant in vitro system, we were able to compare the growth of clinically isolated bacterial biofilms on commercially available K-wires of different materials. 


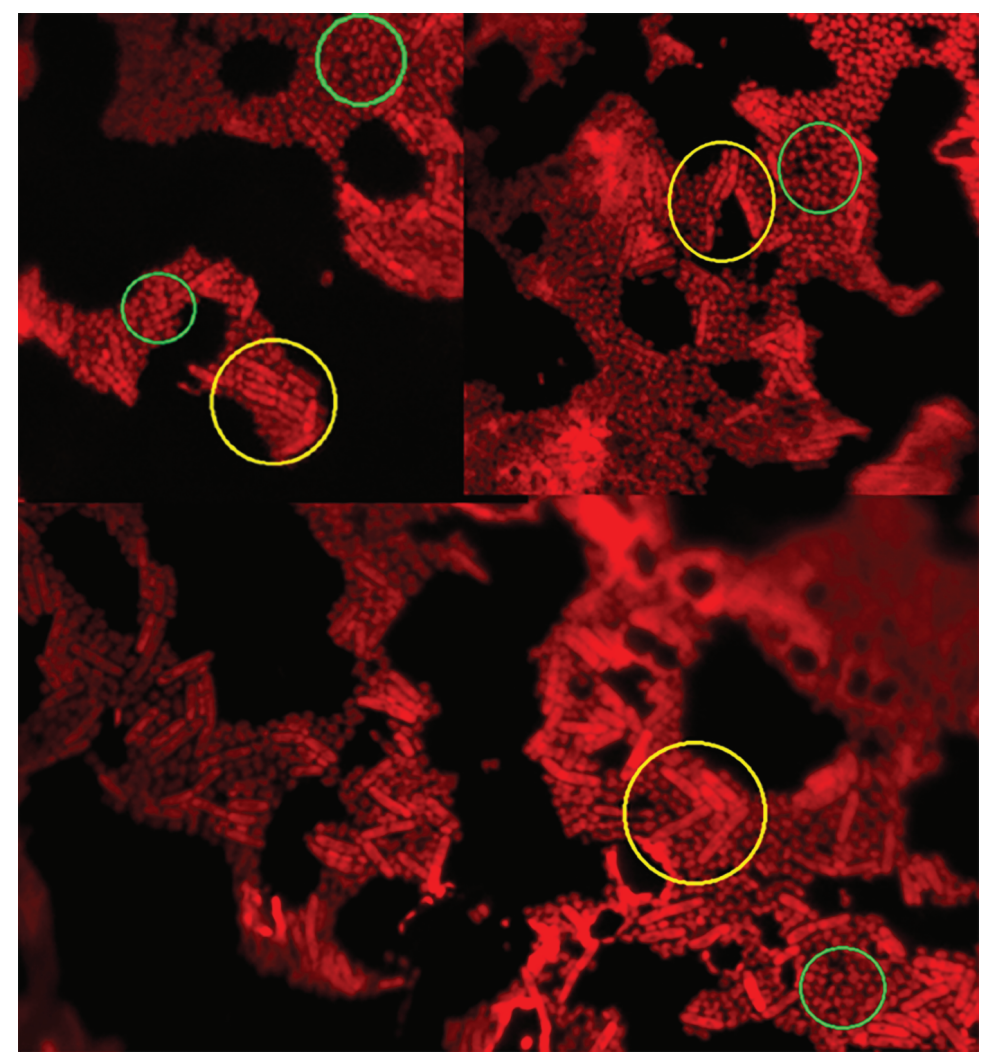

Fig. 5: Epifluorescent 100x images of co-cultured biofilms of S. epidermidis (green circles) and swarming P. mirabilis (yellow circles), grown and imaged on glass coverslips

This study found that $S$. epidermidis and P. mirabilis, two common pin site bacteria, developed biofilms on titanium alloy K-wires with a surface density of viable cells $3.0 \times$ less than on uncoated stainless steel. There is evidence that titanium pins lead to better clinical outcomes than stainless steel pins. ${ }^{19-21}$ Our results suggest that one of the reasons for this commonly observed superiority might be the greater resistance to bacterial biofilm growth on titanium and thus to lower infection rates.

Titanium has been found previously in some studies to resist bacterial adhesion better than stainless stee ${ }^{23,40}$ although other researchers found minimal differences. ${ }^{24,36}$ The mechanisms that underlie the low susceptibility to bacterial adhesion and biofilm growth on titanium might be from a smoother nanostructure and formation of a thick surface oxide layer. ${ }^{41}$ This oxide layer is also thought to improve biocompatibility in vivo which reduces pin loosening-a factor in pin site infection. ${ }^{42}$ The removal of this layer by polishing, as was done in some other studies, may lessen the measured difference in bacterial adhesion between the two metals. ${ }^{24,36}$ However, there is little consensus currently on the effect of the nanostructure or the oxide layer on the biocompatibility and susceptibility to biofilm formation on titanium. ${ }^{16,18,43}$ Although commercially pure titanium and the Ti-6Al-4V alloy used in this work have been found to absorb biomolecules differently using surface chemical techniques, ${ }^{44}$ a recent review of the topic found no evidence that they exhibited different biocompatibilities or susceptibilities to biofilm formation. ${ }^{45}$

S. epidermidis and $P$. mirabilis grew biofilms on hydroxyapatitecoated K-wires to a viable cell surface density that was nonsignificantly greater than on stainless steel and $4.5 \times$ higher than on titanium alloy. Hydroxyapatite has been used as a coating on stainless steel pins to improve osseointegration and reduce pin loosening, although its effects on clinical infection rates are less clear. ${ }^{26,46}$ The few in vitro studies of bacterial adhesion to and biofilm formation on hydroxyapatite coatings have produced conflicting results. Oga et al. found with scanning electron microscopy that S. epidermidis adhered in greater numbers to hydroxyapatite than to the uncoated metals used in this study, ${ }^{47}$ and Ravn et al. have used microcalorimetry to reach a similar conclusion with S. aureus. ${ }^{48}$ In contrast, two other microbiological studies have found that hydroxyapatite is comparatively resistant to staphylococcal adhesion. ${ }^{27,49}$ Our finding that hydroxyapatite exhibited a similar but slightly increased propensity for biofilm formation with respect to stainless steel mirrors the similar clinical infection rate seen with hydroxyapatite and stainless steel. ${ }^{46,50}$ The slightly greater viable cell surface density measured on hydroxyapatite in this work is likely to be because, at least in part, of its greater roughness. ${ }^{48,51}$

In both the microtiter plate assay and the bioreactor, P. mirabilis formed more biofilm than $S$. epidermidis but not significantly more ( $p<0.08$ in the crystal violet assay). This is the first time, to our knowledge, that the biofilm-forming capabilities of these two species have been compared. P. mirabilis's better biofilm formation may be related to its motility which allows it to swarm over implant surfaces. ${ }^{52}$ Co-culture of S. epidermidis and P. mirabilis did not significantly increase biofilm formation beyond single culture of these species. Some other studies have shown a profound difference in biofilm formation between single and mixed species 

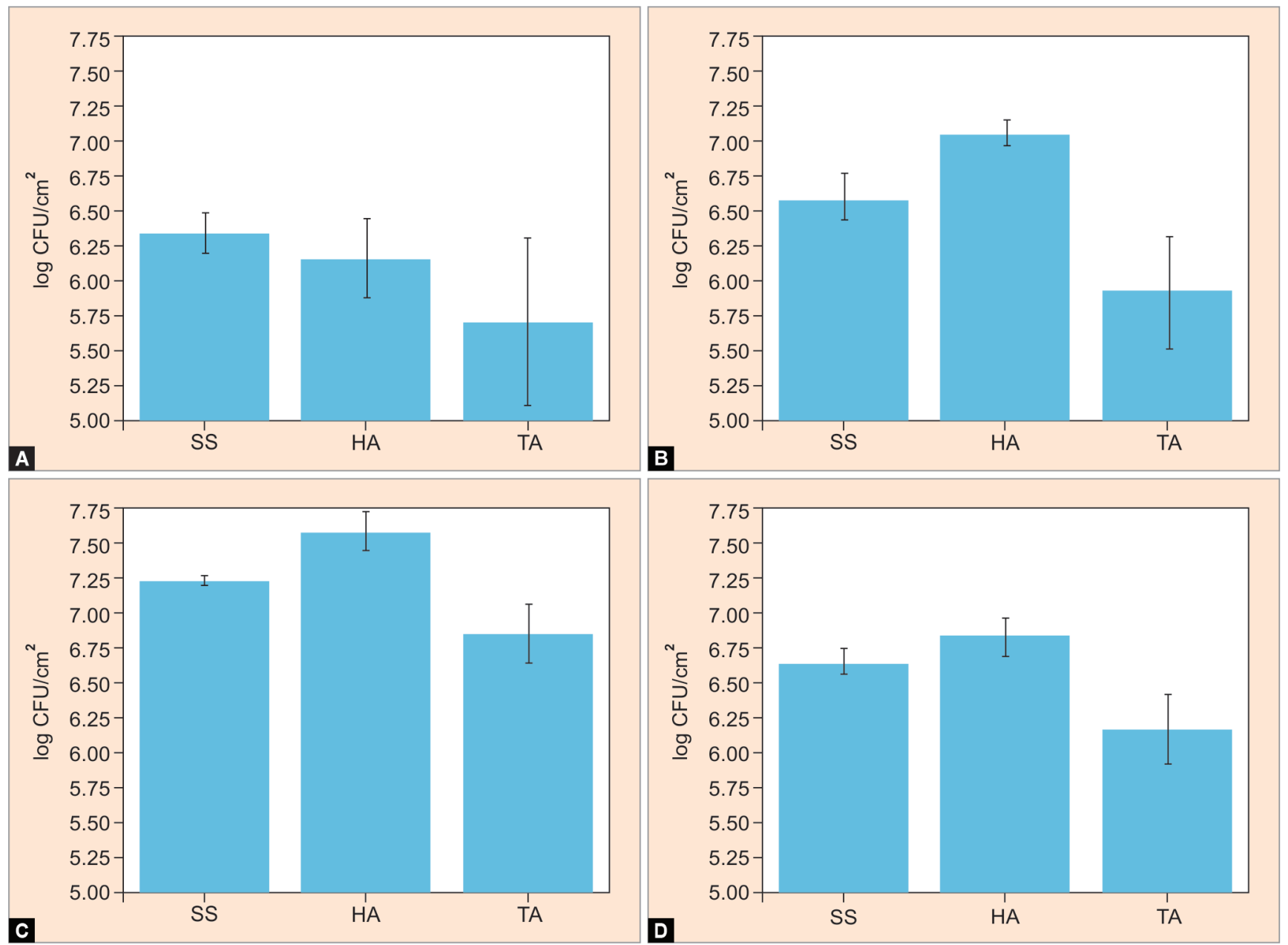

Figs 6A to D: Biofilm growth on K-wires of different materials; SS, stainless steel; HA, hydroxyapatite-coated stainless steel; TA, titanium alloy: (A) S. epidermidis in LB broth; (B) Co-culture of S. epidermidis and P. mirabilis in LB broth; (C) Co-culture of S. epidermidis and P. mirabilis in human serum; (D) Average of all results, both single culture and co-culture. The columns represent the means of the repeats [2-4 repeats in (A)-(C), 8-14 repeats in (D)] with error bars representing \pm standard error

cultures, ${ }^{53}$ very weak biofilm formers can attach to, and become part of, biofilms produced by another species. ${ }^{54}$ However, mixed biofilm interactions are species dependent and may be competitive as well as cooperative. ${ }^{55}$ The results of this study are consistent with observations of biofilms grown on polymethyl methacrylate, indicating that any interactions between $P$. mirabilis and $S$. epidermidis lead to no significant increase in biofilm formation. ${ }^{56}$

The use of pure human serum as a growth medium in the bioreactor significantly increased biofilm formation compared to $10 \%$ diluted LB broth. This is probably a result of the increased nutrient levels in human serum outweighing the recently reported inhibitory effect exerted by serum proteins on biofilm growth of S. epidermidis. ${ }^{57}$

In conclusion, we compared the in vitro biofilm growth of clinical strains of $S$. epidermidis and P. mirabilis on commercially available orthopedic K-wires made from titanium alloy, uncoated stainless steel, and hydroxyapatite-coated steel. These common pin site bacterial species grew as biofilms significantly less well on titanium (as measured by the surface density of viable cells) than on the other two materials. Our results are consistent with the majority of clinical studies which have found that pin site infection rates, relative to those obtained using uncoated stainless steel, are reduced by the use of titanium and more or less unchanged by the use of hydroxyapatite-coated pins. ${ }^{19-21,46,50}$

Although our results are consistent with the majority of clinical studies, they are subject to the limitations of an in vitro study design. ${ }^{58}$ Factors besides bacterial biofilm growth on pins were not investigated, and several such factors are doubtless involved in the development of bacterial infections at clinical pin sites (e.g., nutrient availability, immune response, and local microbiota). Mechanical factors such as wire tension and frame construction, as well as different clinical situations (e.g., nonunion or gradual deformity correction) can also be influential. In vivo studies are needed to measure the relative importance of these factors.

\section{Compliance with Ethical Standards}

\section{Ethical Approval}

All procedures performed in studies involving human participants were in accordance with the ethical standards of the institutional and/or national research committee and with the 1964 Helsinki Declaration and its later amendments or comparable ethical standards. 


\section{Informed Consent}

Informed consent was obtained from all individual participants included in the study.

\section{References}

1. Bible JE, Mir HR. External fixation: principles and applications. J Am Acad Orthop Surg 2015;23(11):683-690. DOI: 10.5435/JAAOS-D-1400281.

2. Fragomen AT, Rozbruch SR. The mechanics of external fixation. HSS J 2007;3(1):13-29. DOI: 10.1007/s11420-006-9025-0.

3. Mock C, Cherian MN. The global burden of musculoskeletal injuries: challenges and solutions. Clin Orthop Relat Res 2008;466(10):23062316. DOI: 10.1007/s11999-008-0416-z.

4. Andruszkow $\mathrm{H}$, Pfeifer R, Horst $\mathrm{K}$, et al. External fixation in the elderly. Injury 2015;46:S7-S12. DOI: 10.1016/S0020-1383(15)30004-8.

5. Kazmers NH, Fragomen AT, Rozbruch SR. Prevention of pin site infection in external fixation: a review of the literature. Strat Traum Limb Recon 2016;11(2):75-85. DOI: 10.1007/s11751-016-0256-4.

6. DeJong ES, DeBerardino TM, Brooks DE, et al. Antimicrobial efficacy of external fixator pins coated with a lipid stabilized hydroxyapatite/ chlorhexidine complex to prevent pin tract infection in a goat model. J Trauma 2001;50(6):1008-1014. DOI: 10.1097/00005373-20010600000006.

7. Antoci $\mathrm{V}$, Ono $\mathrm{CM}$, Antoci $\mathrm{V}$, et al. Pin-tract infection during limb lengthening using external fixation. Am J Orthop 2008;37(9): E150-E154.

8. Ferreira N, Marais LC. Prevention and management of external fixator pin track sepsis. Strat Traum Limb Recon 2012;7(2):67-72. DOI: 10.1007/ s11751-012-0139-2.

9. Bibbo C, Brueggeman J. Prevention and management of complications arising from external fixation pin sites. J Foot Ankle Surg 2010;49(1):87-92. DOI: 10.1053/j.jfas.2009.07.026.

10. Loder RT. The influence of diabetes mellitus on the healing of closed fractures. Clin Orthop Relat Res 1988;232(232):210-216. DOI: 10.1097/00003086-198807000-00028.

11. Brady RA, Calhoun JH, Leid JG, et al. Infections of orthopaedic implants and devices. In: Shirtliff ME, Leid JG. The Role of Biofilms in Device-Related Infections, Springer Series on Biofilms, vol. 3, Berlin: Springer; 2008. pp. 15-55.

12. Ceroni $D$, Grumetz $C$, Desvachez $O$, et al. From prevention of pin-tract infection to treatment of osteomyelitis during paediatric external fixation. J Child Orthop 2016;10(6):605-612. DOI: 10.1007/s11832-0160787-8.

13. Davey ME, O'Toole GA. Microbial biofilms: from ecology to molecular genetics. Microbiol Mol Biol Rev 2000;64(4):847-867. DOI: 10.1128/ mmbr.64.4.847-867.2000.

14. Gristina A. Biomaterial-centered infection: microbial adhesion versus tissue integration. Science 1987;237(4822):1588-1595. DOI: 10.1126/ science.3629258.

15. Hall-Stoodley L, Costerton JW, Stoodley P. Bacterial biofilms: from the natural environment to infectious diseases. Nat Rev Microbiol 2004;2(2):95-108. DOI: 10.1038/nrmicro821.

16. Ploux L, Ponche A, Anselme K. Bacteria/material interfaces: Role of the material and cell wall properties. J Adhes Sci Tech 2010;24(13-14): 2165-2201. DOI: 10.1163/016942410X511079.

17. Campoccia D, Montanaro L, Arciola CR. The significance of infection related to orthopedic devices and issues of antibiotic resistance. Biomaterials 2006;27(11):2331-2339. DOI: 10.1016/ j.biomaterials.2005.11.044.

18. Jennison T, McNally M, Pandit H. Prevention of infection in external fixator pin sites. Acta Biomater 2014;10(2):595-603. DOI: 10.1016/j. actbio.2013.09.019.

19. Clauss M, Graf S, Gersbach S, et al. Material and biofilm load of K wires in toe surgery: titanium versus stainless steel. Clin Orthop Relat Res 2013;471(7):2312-2317. DOI: 10.1007/s11999-013-2919-5.
20. Pieske $\mathrm{O}$, Geleng $\mathrm{P}$, Zaspel J, et al. Titanium alloy pins versus stainless steel pins in external fixation at the wrist: a randomized prospective study. J Trauma Injury Infect Crit Care 2008;64(5):1275-1280. DOI: 10.1097/TA.0b013e31815e40e0.

21. Silvestre MD, Bakaloudis $G$, Lolli F, et al. Late-developing infection following posterior fusion for adolescent idiopathic scoliosis. Eur Spine J 2011;20(S1):S121-S127. DOI: 10.1007/s00586-011-1754-1.

22. Arens $S$, Schlegel U, Printzen $G$, et al. Influence of materials for fixation implants on local infection. J Bone Joint Surg Br 1996;78(4):647-651. DOI: 10.1302/0301-620X.78B4.0780647.

23. Metsemakers WJ, Schmid T, Zeiter S, et al. Titanium and steel fracture fixation plates with different surface topographies: influence on infection rate in a rabbit fracture model. Injury 2016;47(3):633-639. DOI: 10.1016/j.injury.2016.01.011.

24. Shida T, Koseki H, Yoda I, et al. Adherence ability of staphylococcus epidermidis on prosthetic biomaterials: an in vitro study. Int J Nanomed 2013;8:3955-3961. DOI: 10.2147/IJN.S51994.

25. Moroni A, Cadossi M, Romagnoli M, et al. A biomechanical and histological analysis of standard versus hydroxyapatite-coated pins for external fixation. J Biomed Mater Res B Appl Biomater 2008;86(2):417-421. DOI: 10.1002/jbm.b.31036.

26. Patel A, Ghai A, Anand A. Clinical benefit of hydroxyapatite-coated versus uncoated external fixation: a systematic review. Int J Orthop 2016;3(9):581-590. DOI: 10.17554/j.issn.2311-5106.2016.03.163.

27. Arciola CR, Montanaro L, Moroni A, et al. Hydroxyapatite-coated orthopaedic screws as infection resistant materials: in vitro study. Biomaterials 1999;20(4):323-327. DOI: 10.1016/s0142-9612(98)00168-9.

28. A $10 \mu \mathrm{L}$ loop of each colony isolate was grown overnight at $37.5^{\circ} \mathrm{C}$ in 5 $\mathrm{mL}$ LB. Cells were harvested by centrifuging at $13,000 \mathrm{~g}$ for 3 minutes, washing in PBS, and a second centrifugation. The supernatant was discarded and bacteria were incubated first for 2 hours in $1.6 \mathrm{~mL}$ of 10 $\mathrm{mM}$ Tris- $\mathrm{HCl}(\mathrm{pH} 8)$ with $4 \mu \mathrm{g}$ of lysozyme (Sigma-Aldrich), followed by an hour with $25 \mu \mathrm{L}$ proteinase-K (Sigma-Aldrich) and $200 \mu \mathrm{L}$ lysis buffer (50 mM Tris, 100 mM EDTA, 1\% SDS, pH 8.0). Extraction and purification was completed using the DNeasy blood and tissue kit (Qiagen) according to the manufacturer's instructions. Extracted DNA concentration was measured using a Nanodrop ND-1000 spectrophotometer (Thermo Scientific). If extracted isolates yielded a DNA concentration $<30 \mathrm{ng} /$ $\mu \mathrm{L}$ then the extraction process was repeated with $5 \times 15$-second bursts of ultrasonic cell lysis (Microson XL-2000 ultrasonic cell disruptor, power setting 8) immediately after incubation with proteinase-K.

29. Bacterial DNA was amplified using outer primers that targeted the $16 \mathrm{~S}$ gene of all bacteria commonly associated with wound infections [28] and an inner primer that targeted the 16S hypervariable region unique to each species of bacteria. The primers used were: $16 \mathrm{~S}$ Outer Forward (5'-GTG TAG CGG TGA AAT GCG-3'), 16S Outer Reverse (5'-ACG GGC GGT GTG TAC AA-3'), 16SInnerForward (5'-GGT GGA GCA TGT GGT TTA-3'), 16SInnerReverse (5'-CCA TTG TAG CAC GTG TGT-3'). A $50 \mu \mathrm{L}$ master mix was prepared to the following final concentrations: $1 \times \mathrm{Q} 5$ reaction buffer (containing $\mathrm{MgCl}_{2}$ ), $200 \mu \mathrm{M}$ dNTPs, $0.5 \mu \mathrm{M}$ forward primer, $0.5 \mu \mathrm{M}$ reverse primer, $0.02 \mathrm{U} / \mu \mathrm{L}$ Q5 polymerase, $10 \mathrm{ng}$ template DNA. The $\mathrm{nPCR}$ reaction was completed on a Biometra TProfessional Thermocycler.

30. Sauer P, Gallo J, Kesselová M, et al. Universal primers for detection of common bacterial pathogens causing prosthetic joint infection. Biomed Pap Med Fac Univ Palacky Olomouc Czech Repub 2005;149(2):285-288. DOI: 10.5507/bp.2005.043.

31. Altschul SF, Gish W, Miller W, et al. Basic local alignment search tool. J Mol Biol 1990;215(3):403-410. DOI: 10.1016/S0022-2836(05)80360-2.

32. O'Toole GA, Kolter R. Initiation of biofilm formation in Pseudomonas fluorescens WCS365 proceeds via multiple, convergent signalling pathways: a genetic analysis. Mol Microbiol 1998;28(3):449-461. DOI: 10.1046/j.1365-2958.1998.00797.x.

33. Donlan RM, Piede JA, Heyes CD, et al. Model system for growing and quantifying streptococcus pneumoniae biofilms in situ and in real time. Appl Environ Microbiol 2004;70(8):4980-4988. DOI: 10.1128/ AEM.70.8.4980-4988.2004. 
34. Bjerkan G, Witsø E, Bergh K. Sonication is superior to scraping for retrieval of bacteria in biofilm on titanium and steel surfaces in vitro. Acta Orthop 2009;80(2):245-250. DOI: 10.3109/17453670902947457.

35. Herigstad B, Hamilton M, Heersink J. How to optimize the drop plate method for enumerating bacteria. J Microbiol Methods 2001;44(2):121-129. DOI: 10.1016/s0167-7012(00)00241-4.

36. Hudetz D, Ursic Hudetz S, Harris LG, et al. Weak effect of metal type and Ica genes on staphylococcal infection of titanium and stainless steel implants. Clin Microbiol Infect 2008;14(12):1135-1145. DOI: 10.1111/j.1469-0691.2008.02096.x.

37. Battin TJ, Sloan WT, Kjelleberg $S$, et al. Microbial landscapes: new paths to biofilm research. Nat Rev Microbiol 2007;5(1):76-81. DOI: 10.1038/nrmicro1556.

38. Kiedrowski MR, Horswill AR. New approaches for treating staphylococcal biofilm infections. Ann N Y Acad Sci 2011;1241(1): 104-121. DOI: 10.1111/j.1749-6632.2011.06281.x.

39. Lebeaux $D$, Chauhan $A$, Rendueles $O$, et al. From in vitro to in vivo models of bacterial biofilm-related infections. Pathogens 2013;2(2):288-356. DOI: 10.3390/pathogens2020288.

40. Akens MK, Chien C, Katchky RN, et al. The impact of thermal cycling on staphylococcus aureus biofilm growth on stainless steel and titanium orthopaedic plates. BMC Musculoskel Disord 2018;19(1):1-6. DOI: 10.1186/s12891-018-2199-z.

41. Raikar GN, Gregory JC, Ong JL, et al. Surface characterization of titanium implants. J Vac Sci Tech A: Vac Surf Films 1995;13(5): 2633-2637. DOI: 10.1116/1.579462.

42. Chin MYH, Sandham A, de Vries J, et al. Biofilm formation on surface characterized micro-implants for skeletal anchorage in orthodontics. Biomaterials 2007;28(11):2032-2040. DOI: 10.1016/j. biomaterials.2006.12.014.

43. Neoh KG, Hu X, Zheng D, et al. Balancing osteoblast functions and bacterial adhesion on functionalized titanium surfaces. Biomaterials 2012;33(10):2813-2822. DOI: 10.1016/j.biomaterials.2012.01.018.

44. Kerber SJ. Bioreactivity of titanium implant alloys. J Vac Sci Tech A: Vac Surf Films 1995;13(5):2619-2623. DOI: 10.1116/1.579460.

45. Shah FA, Trobos M, Thomsen $P$, et al. Commercially pure titanium (cp-Ti) versus titanium alloy (Ti6Al4V) materials as bone anchored implants - is one truly better than the other? Mater Sci Eng C Mater Biol Appl 2016;62:960-966. DOI: 10.1016/j.msec.2016.01.032.

46. Saithna A. The influence of hydroxyapatite coating of external fixator pins on pin loosening and pin track infection: a systematic review. Injury 2010;41(2):128-132. DOI: 10.1016/j.injury.2009.01.001.
47. Oga M, Arizono T, Sugioka Y. Bacterial adherence to bioinert and bioactive materials studied in vitro. Acta Orthop Scand 1993;64(3):273-276. DOI: 10.3109/17453679308993623.

48. Ravn C, Ferreira IS, Maiolo E, et al. Microcalorimetric detection of staphylococcal biofilm growth on various prosthetic biomaterials after exposure to daptomycin. J Orth Res 2018;36(10):2809-2816. DOI: $10.1002 /$ jor.24040.

49. Alam F, Balani K. Adhesion force of staphylococcus aureus on various biomaterial surfaces. J Mech Behav Biomed Mater 2017;65:872-880. DOI: 10.1016/j.jmbbm.2016.10.009.

50. Pieske O, Pichlmaier L, Kaltenhauser F, et al. Hydroxyapatite-coated pins versus titanium alloy pins in external fixation at the wrist: a controlled cohort study. J Trauma 2011;70(4):845-851. DOI: 10.1097/ TA.0b013e3181e97761.

51. Teughels W, Van Assche N, Sliepen I, et al. Effect of material characteristics and/or surface topography on biofilm development. Clin Oral Implants Res 2006;17(Suppl 2):68-81. DOI: 10.1111/j.16000501.2006.01353.x.

52. Stickler D, Hughes G. Ability of Proteus mirabilis to swarm over urethral catheters. Eur J Clin Microbiol Infect Dis 1999;18(3):206-208. DOI: $10.1007 / \mathrm{s} 100960050260$.

53. Ica T, Caner V, Istanbullu O, et al. Characterization of mono- and mixed-culture Campylobacter jejuni biofilms. Appl Environ Microbiol 2012;78(4):1033-1038. DOI: 10.1128/AEM.07364-11.

54. Cowan MM, Warren TM, Fletcher M. Mixed species colonization of solid surfaces in laboratory biofilms. Biofouling 1991;3(1):23-34. DOI: 10.1080/08927019109378159.

55. Rao D, Webb JS, Kjelleberg S. Competitive interactions in mixedspecies biofilms containing the marine bacterium Pseudoalteromonas tunicata. Appl Environ Microbiol 2005;71(4):1729-1736. DOI: 10.1128/ AEM.71.4.1729-1736.2005.

56. Chang CC, Merritt K. Effect of Staphylococcus epidermidis on adherence of Pseudornonas aeruginosa and Proteus mirabilis to polymethyl methacrylate (PMMA) and gentamicin-containing PMMA. J Orth Res 1991;9(2):284-288. DOI: 10.1002/jor.1100090217.

57. She $P$, Chen L, Qi Y, et al. Effects of human serum and apotransferrin on staphylococcus epidermidis RP62A biofilm formation. Microbiologyopen 2016;5(6):957-966. DOI: 10.1002/ mbo3.379.

58. Roberts AEL, Kragh KN, Bjarnsholt $\mathrm{T}$, et al. The limitations of in vitro experimentation in understanding biofilms and chronic infection. $J$ Mol Biol 2015;427(23):3646-3661. DOI: 10.1016/j.jmb.2015.09.002. 